\section{Custo do transplante hepático no Hospital das Clínicas da Universidade Federal de Pernambuco}

\section{Liver transplantation cost analysis at the Hospital das Clínicas da Universidade Federal de Pernambuco}

Álvaro Antônio Bandeira Ferraz 1 Edmundo Pessoa de Almeida Lopes 2 Marcelo José Antunes Sette 3 Michelle Maria Gonsalves Godoy 4 Maria Helena Oliveira Lins Andrade Lima 5 José Guido Corrêa de Araújo Júnior 6 Balduíno Guedes da Nóbrega Júnior 7 Edmundo Machado Ferraz 8

1 Departamento de Cirurgia da Universidade Federal de Pernambuco. Unidade de Transplantes. Hospital das Clínicas. Av. Prof. Moraes Rego s. n. Cidade Universitária. Recife, PE. Brasil. CEP: $50.670-420$.

2 Departamento de Medicina Clínica da Universidade Federal de Pernambuco

3,4 Unidade de Transplantes do Hospital das Clínicas da Universidade Federal de Pernambuco

5-8 Serviço de Cirurgia Geral do Hospital das Clínicas da Universidade Federal de Pernambuco

\begin{abstract}
Objectives: the aim of this study was to determine the overall cost involving the surgical procedure for liver transplantation performed at the Hospital das Clínicas, Universidade Federal de Pernambuco (HC/UFPE).

Methods: the authors analyzed the last four liver transplantation procedures performed at the mentioned hospital. Patients were classified according to the United Network for Organ Sharing Health Severity Index into class one, (two patients), class two (one patient); and class four (one patient). The parameters evaluated to determine the procedure costs were: hospitalization, operating room and monitoring equipment fees. Cost estimate was provided by the financial department of the hospital in July 2001; and comprised of oxygen fees, disposable material and medications, diagnostic tests and hemotherapy costs. The authors did not include cost analysis for procedure on donors, nor the cost of the medical, nursing and paramedics involved.

Results: the disposable materials items accounted for the leading cost parameters, reaching 48,5\% of the overall cost, followed by hospitalization and medication fees with $17,9 \%$ and $16,1 \%$, respectively.

Conclusions: the authors concluded that the average cost of a liver transplantation at the HC/UFPE in the period 1998-2001, was of $R \$ 22.184,00$ reais (US\$8.873,76; $U \$ 1,00=R \$ 2,50$ ).
\end{abstract}

Key words Liver transplantation, Health care cost, Hepatitis $C$.

\section{Resumo}

Objetivos: estipular o custo do procedimento cirúrgico do transplante de fígado realizado no Hospital das Clínicas da Universidade Federal de Per-

Métodos: foram analisados os primeiros quatro transplantes de fígado realizados no HC/UFPE. Os pacientes foram classificados conforme o índice de gravidade da United Network for Organ Sharing em classe um - dois pacientes; classe dois - um paciente; classe quatro - um paciente. Os custos foram determinados da seguinte maneira: diárias, taxas de sala e aparelhagem - estimativa realizada pelo setor de faturamento do HC/UFPE em julho de 2001; taxa de oxigênio, materiais descartáveis, medicamentos; exames e hemoterapia. Não foram levantados os custos da retirada do fígado, assim como não foram considerados os custos de equipe médica, de enfermagem, técnicos e paramédicos envolvidos no procedimento.

Resultados: o item de materiais descartáveis foi o que mais onerou o transplante, correspondendo a cerca de $48,5 \%$ do custo total, seguido pelas diárias e medicamentos com 17,9\% e 16,1\% respectivamente.

Conclusões: os autores concluíram que o custo médio do procedimento cirúrgico do transplante hepático no HC/UFPE foi no período de 1998 a 2001 de $R \$ 22.184,40$ (US\$8.873,76; U\$1,00 = R\$2,50).

Palavras-chave Transplante de fígado, Custos de cuidados de saúde, Hepatite $C$ nambuco (HC/UFPE). 


\section{Introdução}

Especial atenção tem sido dada às despesas médicohospitalares e, principalmente, às verbas destinadas à saúde pública em todo o mundo. Se levarmos em consideração o Brasil, essa atenção tem merecido uma preocupação ainda maior, ora pela constante e cada vez maior escassez de verbas destinadas à saúde, ora pelo mau uso da mesma. Neste cenário, procedimentos de alto custo e com pouca abrangência populacional são constantemente questionados. $\mathrm{O}$ transplante de fígado exemplifica bem esse cenário. É um procedimento caro, em um país que prima pela falta e mau uso da verba destinada a saúde da população.

Nos Estados Unidos o transplante de fígado tem um custo de cerca de US\$200.000,00 ao final do primeiro ano do transplante. 1,2

Desde 1983, quando os membros da Conferência de Consenso dos Institutos Nacionais de Saúde dos Estados Unidos (NIH), segundo Detre et al. (1996: 824), ${ }^{3}$ declararam que: "Após revisão extensa e consideração de todos os dados disponíveis, este painel conclui que o transplante hepático é uma modalidade terapêutica para doença hepática em fase final que merece aplicação mais ampla", o número de transplantes de fígado, em todo o mundo, tem demonstrado um crescimento surpreendente, não só pelo aumento quantitativo dos transplantes mas principalmente pelos resultados alcançados. Cerca de $90 \%$ dos pacientes transplantados retornam às atividades normais ao final do primeiro ano. ${ }^{2}$

Apesar de já estar consolidado como tratamento efetivo da insuficiência hepática, o transplante hepático ainda traz consigo o estigma de ser muito oneroso e que, em países e regiões subdesenvolvidas, como o Nordeste brasileiro, a sua realização estaria comprometendo políticas preventivas de saúde pública de maior abrangência.

O objetivo deste trabalho foi estipular o custo do procedimento cirúrgico do transplante de fígado realizado no Hospital das Clínicas da Universidade Federal de Pernambuco (HC/UFPE).

\section{Métodos}

Durante o período de maio de 1998 a maio de 2001, foram realizados no Hospital das Clínicas da Universidade Federal de Pernambuco, Brasil, cinco transplantes de fígado.

É importante salientar que foram analisados apenas os transplantes realizados no HC/UFPE, pois grande parte da equipe também realiza transplante no Hospital Memorial São José, onde outros quatro transplantes foram realizados.

Entende-se por custo do procedimento cirúrgico do transplante hepático o custo da cirurgia e do pósoperatório imediato. Foram realizados levantamentos retrospectivos nos prontuários dos pacientes, analisando todos os itens utilizados durante o período compreendido entre o procedimento cirúrgico e a alta ou o óbito. O prontuário do primeiro paciente dentre os cinco transplantados apresentava inúmeras subnotificações e extravio de algumas páginas, que impossibilitaram a análise desse paciente.

As causas da doença hepática e os motivos das indicações dos quatro pacientes analisados foram:

Paciente um Hepatite fulminante (idiopática)

Paciente dois $>$ Cirrose por hepatite C - Hemorragia digestiva

Paciente três > Cirrose por hepatite C - Hemorragia digestiva

Paciente quatro Cirrose por hepatite $\mathrm{C}+$ Hepatocarcinoma

Os pacientes foram classificados conforme o índice de gravidade da UNOS (United Network for Organ Sharing) em classe um (pacientes em casa, com atividades normais) - dois pacientes; classe dois (pacientes necessitando cuidado médico contínuo. Internações de curta duração podem ser necessárias) - um paciente; classe quatro (internação na unidade de terapia intensiva) - um paciente. Dois pacientes já tinham sido submetidos a operações no abdome superior, previamente.

Foram analisados de maneira separadas as diárias hospitalares e de UTI, as taxas de sala, aparelhagem e de oxigênio, os materiais descartáveis, os medicamentos, os exames utilizados e a hemoterapia. Dentre os materiais descartáveis foram analisados em média 75 itens por transplante, enquanto que na análise dos medicamentos foram analisados em média 63 itens.

Os custos foram determinados da seguinte maneira:

Diárias, taxas de sala e aparelhagem - estimativa realizada pelo setor de faturamento do Hospital das Clínicas da UFPE em julho de 2001;

Taxa de oxigênio, materiais descartáveis, medicamentos - valor de compra conseguido através de licitações;

Exames - valor determinado pelo setor de faturamento do HC da UFPE de acordo com os preços de materiais e depreciação de equipamentos;

Hemoterapia - valor reembolsado pelo sistema único de saúde (SUS).

Não foram levantados os custos da retirada do fígado, assim como não foram considerados os custos 
de equipe médica, de enfermagem, pois nos pacientes analisados não houve repasse de honorários à equipe transplantadora.

Também não foram consideradas as despesas com a remuneração dos técnicos e paramédicos envolvidos no procedimento.

\section{Resultados}

O tempo de permanência hospitalar dos quatro pacientes foi de $18,6,13$ e 13 dias, respectivamente. Os dois primeiros pacientes foram a óbito. O primeiro paciente transplantado, decorrente de uma hepatite fulminante, apresentou recuperação pós-operatória satisfatória, quando no $17^{\circ}$ dia de pós-operatório, na enfermaria, apresentou fibrilação atrial que não reverteu à cardioversão. O segundo paciente foi a óbito no sexto dia de pós-operatório com o diagnóstico de pulmão de choque e insuficiência renal. Os outros dois pacientes obtiveram alta no $13^{\circ}$ dia de pósoperatório sem nenhuma complicação e até a presente data encontram-se clinicamente bem.

O primeiro paciente analisado, apresentava-se no momento do transplante, em coma grau III e distúrbio severo da coagulação, o que determinou a transfusão de 82 unidades de plasma fresco, 64 unidades de plaquetas e 43 unidades de concentrado de hemácias. O custo total desse paciente foi de $\mathrm{R} \$ 30.387,58$.

Os pacientes três e quatro não apresentaram qualquer complicação e obtiveram alta no $13^{\circ}$ dia de pós-operatório. Ambos os pacientes apresentaram custo de cerca de $\mathrm{R} \$ 19.000,00$, para cada transplante.

No Quadro 1 estão expostos alguns dos valores determinados para o cálculo do custo total do transplante hepático no Hospital das Clínicas da UFPE. 
Valores em reais de alguns itens analisados no custo do transplante de fígado em pacientes no Hospital das Clínicas da Universidade Federal de Pernambuco, Brasil, 1998 a 2001.

\begin{tabular}{|c|c|}
\hline & Preço unitário (R\$) \\
\hline \multicolumn{2}{|l|}{ Diárias } \\
\hline Diária hospitalar & 67,94 \\
\hline Diária UTI & 614,26 \\
\hline \multicolumn{2}{|l|}{ Taxa de sala } \\
\hline Taxa de sala até três horas & 30,70 \\
\hline Hora subseqüente & 10,23 \\
\hline \multicolumn{2}{|l|}{ Taxas de aparelhagem } \\
\hline Monitor & 2,57 \\
\hline Oxímetro & 2,58 \\
\hline Capnógrafo & 7,92 \\
\hline Aspirador & 24,26 \\
\hline Bisturi elétrico bipolar & 15,79 \\
\hline Bomba de infusão & 22,81 \\
\hline \multicolumn{2}{|l|}{ Taxa de oxigênio } \\
\hline Oxigênio (quatro horas) & 3,11 \\
\hline \multicolumn{2}{|l|}{ Exames } \\
\hline Glicose, Uréia e Creatinina & 1,85 (cada) \\
\hline \multicolumn{2}{|l|}{ Sódio, Potássio, Cloreto, } \\
\hline \multicolumn{2}{|l|}{ Amilase, Bilirrubinas, } \\
\hline TGO, TGP & 2,01 (cada) \\
\hline Gasimetria & 15,65 \\
\hline \multicolumn{2}{|l|}{ Hemograma, Fibrinogênio, } \\
\hline TPAE, $\mathrm{Ht} / \mathrm{Hb}$ & 4,11 (cada) \\
\hline Dosagem de Ciclosporina & 4,11 \\
\hline \multicolumn{2}{|l|}{ Hemoterapia } \\
\hline Plasma fresco & 13,74 \\
\hline Concentrado de hemácias & 13,74 \\
\hline Concentrado de plaquetas & 13,74 \\
\hline Crioprecipitado & 13,74 \\
\hline
\end{tabular}

O custo médio do transplante de fígado no Hospital das Clínicas da UFPE foi de R $\$ 22.184,40$. O item de materiais descartáveis foi o que mais onerou o transplante, correspondendo a cerca de $48,5 \%$ do custo total, seguido pelas diárias e medicamentos com $17,9 \%$ e $16,1 \%$ respectivamente. A discriminação dos valores dos transplantes estão expostos na Tabela 1. 
Custos em reais do transplante de fígado por pacientes no Hospital das Clínicas da Universidade de Pernambuco, Brasil, de 1998 a 2001.

\begin{tabular}{|c|c|c|c|c|c|c|}
\hline Itens analisados & Paciente 1 & Paciente 2 & Paciente 3 & Paciente 4 & Total & Média \\
\hline \multicolumn{7}{|l|}{ Diárias } \\
\hline Hospitalar & 203,82 & 135,88 & 747,34 & 747,34 & $1.834,38$ & 917,19 \\
\hline UTI & $9.213,90$ & $2.457,04$ & $1.228,52$ & $1.228,52$ & $14.127,98$ & $3.531,99$ \\
\hline Total & $9.417,72$ & $2.592,92$ & $1.975,86$ & $1.975,86$ & $15.962,36$ & $3.990,59$ \\
\hline Taxa de sala & 184,20 & 225,13 & 133,03 & 122,80 & 665,16 & 166,29 \\
\hline Taxa aparelhagem & $1.366,74$ & $1.822,32$ & 987,09 & 911,16 & $5.087,31$ & $1.271,83$ \\
\hline Taxa oxigênio & 55,98 & 74,64 & 40,43 & 37,32 & 208,37 & 52,09 \\
\hline Descartáveis & $10.755,15$ & $10.768,20$ & $10.755,15$ & $10.752,70$ & $43.031,20$ & $10.757,80$ \\
\hline Medicamentos & $4.644,76$ & $3.071,99$ & $2.889,21$ & $3.742,15$ & $14.348,11$ & $3.587,03$ \\
\hline Exames & $1.228,77$ & 447,33 & 447,33 & 441,67 & $2.565,10$ & 641,27 \\
\hline Hemoterapia & $2.734,26$ & $1.813,68$ & $1.813,68$ & 508,38 & $6.870,00$ & $1.717,50$ \\
\hline Total & $30.387,58$ & $20.816,21$ & $19.041,78$ & $18.492,04$ & $88.737,61$ & $22.184,40$ \\
\hline
\end{tabular}

\section{Discussão}

Não se pode analisar o custo do transplante de fígado usando apenas os números frios que ele determina. O transplante de fígado proporciona uma melhoria global do serviço prestado pela Instituição, tais como melhoria no manuseio do paciente cirúrgico, da anestesia, no conhecimento de metabologia, na qualificação técnica dos cirurgiões e nos conhecimentos de imunossupressão e infecção.

$\mathrm{O}$ transplante de fígado tem deixado de ser encarado como um procedimento de alto custo e pouco retorno, para ser visto como uma fonte de renda às Instituições, determinando inclusive uma corrida às Centrais de Transplantes e ao Ministério da Saúde para credenciar novos centros transplantadores.

De acordo com a tabela de repasse do Sistema Único de Saúde (SUS), o transplante de fígado remunera à Instituição $\mathrm{R} \$ 51.899,46$, acrescidos $\mathrm{R} \$ 900,00$ de procedimento anestésico. Considerando o custo médio deste estudo de $\mathrm{R} \$ 22.184,40$ e mais $\mathrm{R} \$ 4.819,50$ destinados à equipe médica, haveria um lucro de cerca de $\mathrm{R} \$ 24.000,00$, por transplante realizado.

É importante, porém, que seja enfatizado que alguns itens deste trabalho estão subfaturados. O fato de Pernambuco dispor de um hemocentro sem fins lucrativos e de a cobrança dos hemoterápicos não ter sido realizada, diminui sobremaneira esta despesa em especial. Coelho et al.,4 analisando o custo dos transplantes de fígado no Hospital das Clínicas do Paraná, encontraram um custo médio dos hemoderivados de
US\$7.760,50.

O custo do transplante de fígado no Hospital das Clínicas da Universidade Federal do Paraná foi de US $\$ 21.505,53.4$ No entanto, algumas peculiaridades de avaliação de custos devem ser analisadas. $\mathrm{O}$ item que mais onerou o transplante foi o de hemoderivados, com cerca de $30 \%$ do custo total do transplante, seguido pelo custo de medicamentos, com cerca de $20 \%$ do custo total. Os custos dos exames complementares, dos hemoderivados e dos procedimentos foram determinados com base na quantidade de $\mathrm{CH}$ (coeficiente de honorário) estabelecido na tabela de honorários médicos de 1992 da Associação Médica Brasileira. Os custos da diária hospitalar, de UTI e taxa de uso do centro cirúrgico foram baseados nos valores cobrados, pelo hospital, de pacientes de convênio internados em apartamentos e os custos de medicamentos baseados no Índice Brasileiro de Guia Farmacêutico (Brasíndice).

Determinou-se o custo do transplante de fígado no Hospital das Clínicas exatamente como ele o foi, ou seja, utilizando os valores de compra do hospital e dos benefícios que lhe são peculiares por conta de ser um hospital Universitário. O custo varia entre hospitais, de acordo com a natureza da instituição (privada ou pública). A política de compras e cobranças dos hospitais públicos e privados determina uma grande variação do custo de medicamentos, materiais descartáveis e exames, porém este valor, com certeza deverá, servir de base para a implantação de programas de transplante de órgãos em instituições públicas. O custo total do transplante hepático pre- 
cisa incluir na preparação pré-operatória, o acompanhamento tardio e as mais diversas remunerações que uma equipe multidisciplinaar envolve.

O transplante de fígado é um procedimento complexo e caro, como também o é o tratamento conservador do paciente hepatopata crônico sem transplante. Williams et al. 5 verificaram que o tratamento de suporte de pacientes com indicação de transplante hepático envolveu um custo de US $\$ 45.643,00$, quase a metade do custo do transplante hepático (US\$92.866,00) realizado pelo mesmo grupo. Os Estados Unidos gastam, por ano, cerca de US\$514 milhões de dólares em 26.700 hospitalizações e 2.600 mortes por hepatite $\mathrm{C}$, e outros US $\$ 1,8$ bilhão com 101.200 hospitalizações e 13.400 mortes decorrentes de doença hepática induzida por álcool. 6

Em adição, deve-se levar em consideração que tanto a sobrevida quanto a qualidade de vida desses pacientes são bem superiores nos pacientes submetidos a transplante hepático, quando comparados àqueles submetidos ao tratamento conservador. $5,7,8$ No estudo publicado por Williams et al., $582 \%$ dos pacientes transplantados retornaram às suas atividades profissionais ao final do primeiro ano póstransplante.

Cabe mais uma vez alertar que o transplante de fígado é um procedimento caro e complexo. O que parece ser lucro fácil e iminente pode se tornar um grande prejuízo e uma grande decepção. O transplante hepático só deve ser realizado em centros de excelência e por equipes multidisciplinares, altamente qualificadas e treinadas, capazes de lidar com pacientes transplantados e portadores de falência hepática. Não adianta apenas realizar o transplante de fígado, o procedimento puramente técnico. Os centros credenciados devem estar preparados e dispostos a tratar o paciente candidato ao transplante, tanto no pré como no pós-operatório. Esses tratamentos nem sempre remuneram de maneira adequada a instituição, mas o compromisso com o paciente, seus familiares, e com a ética médica não permitem que complicações e intercorrências sejam tratadas em outros estabelecimentos ou por outras equipes.

Em suma, o custo total do transplante hepático apresentado pelos autores difere bastante dos valores norte-americanos e europeus, assim como é inferior aqueles apresentados por Coelho et al. ${ }^{4} \mathrm{em}$ um hospital brasileiro. 1,3,4,9,10 Os autores concluíram que o custo médio do procedimento cirúrgico do transplante hepático no Hospital das Clínicas da UFPE no período de 1998 a 2001 foi de $\mathrm{R} \$ 22.184,40$ o que equivale a $\mathrm{US} \$ 8.873,76(\mathrm{U} \$ 1,00=\mathrm{R} \$ 2,50)$.

\section{Referências}

1. Russell OS. Understanding resourse use in liver transplantation. Jama 1999; 281: 1431-2.

2. Gilbert JR, Schoenfeld DA, Rubin RH, Delmonico FL, Cosi$\mathrm{mi} \mathrm{AB}$. Envolving treds in liver transplantation: an outcome and charge analysis. Transplantation 1999; 67: 246-53.

3. Detre KM, Belle SH, Beringer KC, Bost JE, Daly P. Overall national results of liver transplantation between 1987 and 1991. In: Busutil RW, Klintmalm GB. Transplatation of the liver. Pennsylvania: WB Saunders; 1996. p. 82434.

4. Coelho JCU, Wiederkehr, JC, Lacerda MA, Campos ACL, Zeni Neto C, Matias JEF, Campos GMR. Custo do transplante hepático no Hospital de Clínicas da Universidade Federal do Paraná. Rev Assoc Med Bras 1997; 43: 53-7.

5. Williams JW, Vera S, Evans LS. Socioeconomic aspects of hepatic transplantation. Am J Gastroenterol 1987; 82: 1115-9.
6. Kim WR, Gross JB, Poterucha JJ, Locke GR, Dickson ER. Outcome of hospital care of liver disease associated with hepatites C in the United States. Hepatology 2001; 33: 201-6.

7. Bonsel GJ, Klompmaker IJ, Essink-Bot ML, Habbema JDF, Slooff MJH. Cost effectiveness analysis of the dutch liver transplantation programme. Transplant Proc 1990; 22: 1481-4.

8. Van Thiel DH, Tarter R, Gavaler JS, Potanko WM, Schade, RR. Liver transplantation in adults an analysis of costs and benefits at the University of Pittsburgh. Gastroenterology 1986; 90: 211-6.

9. Eckhoff DE, McGuire BM, Young C, Sellers MT, Contreras JL, Frenette LR, Hudson SL, Bynon JS. Liver transplantation in the era of cost constraints. South Med J 2000; 93: 392-6.

10. O’ Grady JG. Clinical economics review: liver transplantation. Aliment Pharmacol Ther 1997; 11: 445-51.

Recebido em 10 de outubro de 2001

Versão final reapresentada em 23 de julho de 2002

Aprovado em 13 de agosto de 2002 